\title{
Utilizando Sistemas Recomendadores para Predecir Ratings en TV
}

\section{Using Recommender Systems to Predict TV Ratings}

\begin{abstract}
Resumen: En este trabajo se presenta un método que, utilizando sistemas recomendadores, predice ratings de TV a partir de los perfiles de los telespectadores recibidos desde sus receptores de televisión digital. La predicción está basada en un recomendador de contenidos audiovisuales, aplicado sobre grupos de televidentes, que utiliza clasificaciones multidimensionales extraidas de la norma TV-Anytime. Se presentan dos aplicaciones ilustrativas, con el objetivo común de maximizar la calificación en dos contextos distintos: un primer algoritmo para configurar la parrilla de programación tiempo real entre un conjunto de contenidos cuál de ellos debe ser transmitido en un momento determinado por una estación de televisión para maximizar su audiencia inmediata. Palabras-clave: TV Digital, TV-Anytime, sistemas recomendadores, recomendaciones para grupos, predicción de audiencias.
\end{abstract}

Abstract: This paper presents a method for predicting television audience from the profiles of the viewers received rom their digital television receivers. The prediction is based

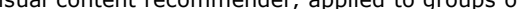
viewers using multidimensional classifications from the TVAnytime standard. We present two sample applications, with the common goal of maximizing the score in two different contexts: a first algorithm to set the weekly program schedule of a television station, and the second one that decides in real time between a set of contents which one must be transmitted at a precise moment by a television station to maximize their immediate audience.

Keywords: Digital TV, TV-Anytime, recommender systems, recommendations for groups, audience prediction.

SOTELO, Rafael; GIL SOLLA, Alberto. Utilizando Sistemas Recomendadores para Predecir Ratings en TV. v. 14, n. 1, p. 11-27, jan./jun. 2011.
Rafael Sotelo

Universidad de Montevideo

Alberto Gil Solla

Universidad de Vigo

\section{Introducción}

L a tecnología ofrece a diario más y más servicios y funcionalidades al ser humano. Estos van desde la posibilidad de suplir incapacidades físicas o intelectuales, hasta la de curar enfermedades crónicas; desde trasladarse eficazmente dentro de una ciudad, a través del globo, o aun fuera de él, hasta la optimización de la producción de alimentos saludables. En particular, a través de los medios de comunicación e Internet se ofrece al individuo la posibilidad de formarse, informarse y entretenerse. Esta oferta crece cotidianamente debido al uso de nuevos medios o a la mejora de otros existentes, otorgándoseles mayor capacidad.

Sin embargo, muchas veces el telespectador se ve desbordado por esta cantidad de información, la cual percibe como una sobrecarga. De esta forma, debe realizar una selección de la información que le interesa, sea ésta, por ejemplo, noticias, contenidos audiovisuales, ofertas comerciales, cursos de formación o de educación. Es así que durante la década de los noventa comenzaron a desarrollarse sistemas recomendadores, que buscan simplificar la labor del individuo, circunscribiendo sus alternativas a las que se estima serán de su interés. Esto se 
consigue haciendo un uso combinado de múltiples fuentes de información (las descripciones de los contenidos, las preferencias de los telespectadores, sus valoraciones, sus consumos históricos...), cuyo peso depende fuertemente de la estrategia empleada por el motor de recomendación.

Estos sistemas están basados en una gran diversidad de técnicas, en las que predominan aquellas que emplean las descripciones para realizar comparaciones meramente sintácticas. Si bien estos enfoques consiguen ofrecer recomendaciones parcialmente acertadas, se restringen a buscar la ocurrencia de las mismas palabras textuales, o con la misma raíz, lo que ha hecho que sus resultados sean limitados. Su rendimiento inicial ha sido mejorado por la aparición de nuevas técnicas, entre las que destacamos aquí el uso de ontologías, las cuales, permitiendo organizar los elementos que describen los contenidos en una estructura jerárquica, habilitan a realizar razonamientos de naturaleza semántica que posibilitan encontrar nuevas relaciones entre los contenidos y los telespectadores. AVATAR, Blanco et al. (2006), es un ejemplo de este enfoque, un recomendador de contenidos audiovisuales para individuos, que utiliza esta clase de técnicas, y para el cual se desarrolló una ontología sobre el dominio de la televisión, una jerarquía descriptiva e interrelacionada de los géneros de la televisión.

Desde la pasada década, los recomendadores han experimentado una extensión de dominio, no orientándose exclusivamente a un telespectador, sino ahora también a grupos de ellos. Si bien esto era un paso lógico, dado que muchas de las actividades de formación, información o entretenimiento que realiza un individuo las lleva a cabo en compañía de otros seres humanos, también es cierto que este tipo de recomendaciones es notablemente distinta a la recomendación individual, ya que ahora no sólo se deben tener en cuenta los intereses de una persona, sino de varias en conjunto, lo que nos obliga a estudiar cómo compatibilizar las preferencias de las partes, priorizando ciertas consideraciones al hacerlo.

En Sotelo et al. (2009) presentamos dos contribuciones. La primera de ellas fue la extensión de la ontología de los contenidos audiovisuales a una de múltiples jerarquías, que será descrita en mayor detalle más adelante. De esta forma, se obtienen recomendaciones más precisas, mejorando incluso las que van dirigidas a un único telespectador. Estas jerarquías, que están basadas en esquemas de clasificación del estándar TV-Anytime, describen el contenido desde ángulos complementarios tales como su género, su formato, la intención con la que fue creado, y su audiencia objetivo. La segunda contribución, dado que frecuentemente se mira televisión en grupos, ya sea en familia o con amigos, está orientada a satisfacer necesidades de la vida real. La misma consistió en el desarrollo de un recomendador de contenidos audiovisuales para grupos de televidentes, además de individuos. El algoritmo de recomendación obtenido discrimina entre grupos homogéneos (aquellos con telespectadores con perfiles similares, típicamente un grupo de amigos) y grupos heterogéneos (los que constan de individuos con perfiles disímiles, por ejemplo una familia).

En el presente artículo, presentamos una nueva contribución que se enmarca en el campo de la predicción de la audiencia de programas televisivos. Extendiendo el foco del problema anterior, modelamos a la audiencia como un grupo, y le aplicamos las técnicas de recomendación para grupos con algunas consideraciones especiales. Con ello conseguimos una predicción de la audiencia que obtendrá un programa, aplicado a dos problemas de enorme significación económica y social. Uno de ellos es la confección de la programación de la parrilla semanal de una emisora de televisión, con el objetivo de maximizar la audiencia que obtendrá. El otro, en cambio, apunta a un fenómeno de la industria de la televisión, relacionado con el vértigo de la emisión en vivo de un programa, que lleva a un problema de tiempo real: el cambio del contenido de un programa mientras se emite, considerando lo emitido por la 
competencia, buscando maximizar la audiencia. Presentaremos una herramienta para elegir cómo cambiar inmediatamente un programa para que la mayoría de la audiencia propia siga fiel y poder captar el máximo posible de la audiencia de la competencia.

\section{El Rating y el Share en TV}

La televisión se financia por la publicidad, la que a su vez está íntimamente relacionada con la cantidad de televidentes que tenga un determinado programa. Cuanto mayor sea numéricamente la audiencia de un programa, más atractivo será para los anunciantes, y representará mayores ingresos para la estación de televisión.

En la medición tradicional de audiencia de televisión se obtienen dos indicadores básicos sobre la audiencia de un programa: el Rating (que indica cuánta gente de todo el universo posible vio el programa) y el Share (que indica qué porcentaje de la gente que estaba viendo televisión durante la emisión del programa, lo vio).

\subsection{Midiendo audiencias en Televisión}

Los radiodifusores quieren maximizar la audiencia, para maximizar el interés de los anunciantes en publicitar en su pantalla. Esta es su principal fuente de ingresos, y por eso deben de tener mayor audiencia que su competencia.

La medición de audiencia de televisión es, por tanto, una tarea muy importante, cuyos principales consumidores son los canales de televisión y las agencias de publicidad. Los canales de televisión, basándose en estas mediciones, toman importantes decisiones sobre su programación. De la misma forma, las agencias de publicidad y los anunciantes modulan la distribución de su inversión publicitaria empleando esos datos como criterio principal. En general, es utilizada como:

i. Una herramienta de diagnóstico para conocer la acogida que ha tenido la programación.

ii. Un instrumento de predicción para estimar futuros resultados en audiencia. Aquí podemos distinguir tres posibles aspectos sobre los que incide, todos de vital importancia para la estación de televisión:

- En qué horario, día de la semana o época del año transmitir contenidos ya adquiridos o producidos.

- Qué clase de nuevos contenidos adquirir.

- Qué clase de nuevos contenidos producir.

iii. Un instrumento de tasación. Según el nivel de audiencia de un programa, así será el valor del tiempo de su publicidad.

iv. Un instrumento de planificación, para decidir qué programas deben seguir emitiéndose, y cuáles eliminarse o modificarse.

Hay distintos métodos de evaluar la audiencia, en los que una muestra permanente de hogares participa en la encuesta. El principal dispositivo utilizado es el people meter, que se conecta a todos los televisores de la muestra panel, los cuales registran cada cierto intervalo el consumo de televisión. Registra automáticamente el encendido, cambios de canal y los miembros del hogar que están expuestos a la televisión a lo largo del día.

La descarga de la información tradicionalmente era realizada por la noche, vía módem telefónico, pero actualmente se está popularizando el uso de otras tecnologías (como ADSL, cable módem, o módem 3G o GPRS) que permiten contar con los datos casi en tiempo real.

El sistema RealTimeWeb descrito en Crivellaro et al. (2008), constituye un sistema de medición de audiencia en tiempo real que presenta sus resultados en una página web accesible a los clientes por diversos medios. Presenta los ratings actuales e históricos de las emisoras de televisión locales que se actualizan cada minuto, e incluye imágenes de lo que la gente estaba viendo en ese momento, renovadas cada 10 segundos. El proceso se demora un minuto ya que recoge los datos de cientos de hogares, los procesa, validándolos y ponderándolos, realiza el cálculo de audiencia y finalmente lo almacena en la base de datos de audiencia. Sistemas como el descrito habilitan a los 
radiodifusores a recibir mediciones de audiencia en tiempo real para varias emisoras de televisión y a identificar rápidamente los cambios del público al trasladarse de una emisora a otra, permitiendo presumir las razones que lo llevaron a hacerlo. De esta forma, el radiodifusor puede tomar decisiones durante la emisión de programas en vivo, realizando acciones que no sería posible llevar a cabo si recibiera dichas mediciones al día siguiente.

En Álvarez et al. (2009), se presentó un sistema futurista que realiza el modelado de la medición de audiencia para redes de radiodifusión e IPTV convergentes. Describe un sistema completo de medición de audiencia convergente, enfocado en IPTV, pero que cubre también radiodifusión terrestre, por cable, por satélite y móvil. Adicionalmente, el consumo del telespectador se modela y se proveen métricas para realizar perfiles de consumo de contenido por parte de los telespectadores.

\subsection{Características de la audiencia televisiva}

Una vez obtenidos los datos de la medición de audiencia se procesan y clasifican en una serie de variables o categorías de clasificación de telespectadores, infiriéndose comportamientos según el sexo, edad, condición socioeconómica, según la fecha del año, el día de la semana y el horario. Finalmente, se asocian con variables relacionadas con el contenido como, por ejemplo, el género del programa, el programa en sí y el canal.

Habitualmente se generan gráficos que marcan claras características del consumo de televisión en función de variables sociodemográficas y de distribución horaria. En la Figura 1 se ven los cinco horarios en que se clasifica el día según la audiencia de televisión: Matinal, Mediodía y Sobremesa, Vespertino, Horario Central (Prime Time) y Trasnoche. Claramente se observa como el Prime Time tiene el máximo de audiencia, y será, por tanto, el que genere mayores ingresos a la emisora, y en donde ésta pondrá sus programas más valiosos.

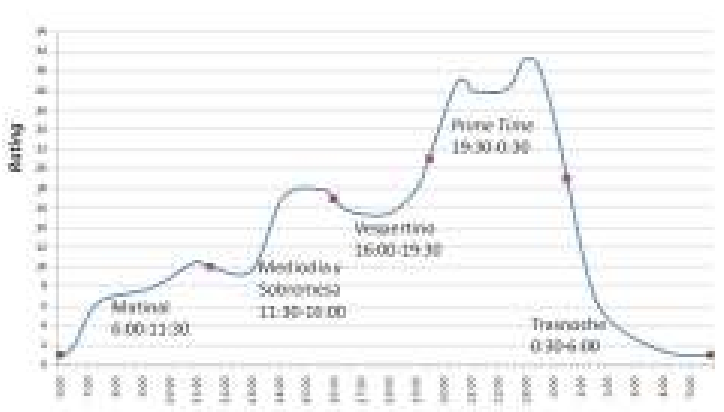

Figura 1 - Evolución Diaria de Rating Total promedio anual.

Las fronteras horarias y el número de franjas varían de país en país, adaptándose a las características particulares de cada mercado. Incluso en un mismo país, distintos analistas pueden manejar distintas clasificaciones. También resulta habitual el realizar gráficas similares en las que se aprecia el rating por horario según una clasificación en particular, por ejemplo, la de la edad del televidente 0 el nivel socioeconómico.

Algunos autores como Gensch et al. (1980), llegan a la conclusión de que el número de televisores encendidos depende más de la época del año, el día de la semana y el horario que del contenido de los programas en sí. Es decir, que en términos generales, si consideramos el rating acumulado sumando el de todas las emisoras de un mismo mercado en un momento dado, encontraremos que es independiente de los contenidos emitidos. Por esto es de vital importancia, para maximizar la audiencia, el rol de quien arma la grilla o parrilla de programación, a fin de conseguir un mayor share, atrayendo la audiencia disponible hacia su canal, quitándosela a la competencia. Esto lo hace basándose en los estudios antedichos, apoyado con herramientas informáticas y en su experiencia y conocimiento del mercado.

\subsection{La programación de una estación de televisión}

La programación, en un sentido amplio, representa la planificación global de la actividad de la emisora cuyo resultado es la emisión de programas, o, en un sentido restringido, la distribución y ordenación de 
espacios en tiempos determinados. El gerente de programación debe definir la audiencia a cubrir, valorar los programas disponibles, seleccionar entre los programas aquellos que mejor se adecuen a la audiencia previamente definida y combinarlos en la parrilla de programación. Existe una estrategia de lucha por el share llamada contraprogramación, que consiste en pretender sorprender a las demás emisoras con emisiones imprevistas, que se programan simultáneamente a los programas de la competencia para intentar capturar parte de su audiencia. Una variante de la contraprogramación se da durante la emisión de un programa, cuando el productor del mismo, siendo consciente de lo que está emitiendo la competencia, toma decisiones sobre el contenido de su programa, variándolo. Puede decidir en qué momento se va a los anuncios publicitarios, cambiar la duración de los bloques del programa o alterar la intervención de un entrevistado. Esto se ve incrementado con modernos dispositivos de medición de audiencia en tiempo real, como el descrito en Crivellaro et al. (2008), que permiten no sólo ver qué están emitiendo las emisoras competidoras, sino además conocer su resultado en rating y en share.

La herramienta principal que tiene el encargado de programación de una emisora de televisión es la medición de audiencia. En ella solamente se registra el número de televidentes que vio el programa, y no se recibe una calificación de los mismos, es decir, no se sabe si el programa es de interés o no de los televidentes. La programación se realiza en base a ciertos presupuestos de conductas de los diferentes grupos socioeconómicos. Se debe conocer en profundidad a la audiencia, y cuanto más precisa sea la información que se tenga, mejor será la configuración de la programación.

Sin embargo, el gran énfasis dado a los números y cálculos de medición de audiencia demuestra el hecho de que las emisoras de televisión se mantienen en una constante búsqueda de la forma de llegar a su audiencia. Pero se debe destacar que en última instancia hay una significativa falta de control. Incluso los ejecutivos de la industria de la televisión norteamericana dependen tanto de la ciencia como de su intuición. Scott Siegler, ex vicepresidente de CBS para desarrollo de drama, lo ha manifestado de este modo:

"Because it is a mass audience - it's an unimaginable large audience - the audience test are so diffused and so general that you've got to be guessing. You can work off precedents about what's worked on television before. You can work off whatever smattering of sociological information you gleaned from whatever sources. You can let your personal judgements enter into it some extents. ...But you never really know.", (ANG, 1991).

A este respecto, creemos que hay ciertas características que son mejorables en los métodos de predicción de audiencias tradicionales:

1. Estos sistemas registran "quiénes ven", "qué ven", "cuándo ven" y "durante cuánto tiempo ven". Sin embargo, no hay posibilidad de ningún tipo de enfoque cualitativo, por ejemplo, que el telespectador indique una calificación para lo que vio.

2. Se infieren las preferencias del televidente por los géneros de los contenidos a partir de sus características de sexo, edad, ocupación, nivel socioeconómico. No se exploran las preferencias de los televidentes en los distintos géneros de programas, y mucho menos en otras características tales como actores, ciudades o períodos de tiempo en que se desarrolla la acción.

3. Existe una fuerte dependencia en la intuición de los gerentes de programación al decidir qué programa funcionará.

En las siguientes secciones presentaremos contribuciones para mejorar este campo, valiéndonos de la tecnología que estimamos que estará disponible a nivel de electrónica de consumidor, tal como prevé TV-Anytime, y utilizando las técnicas de predicción para grupos, aplicándoselas a la audiencia vista como un grupo de individuos de los cuales se dispondrán de los perfiles de telespectador. Predeciremos la audiencia que tendrá un programa en función del interés que despierta en el grupo el contenido que ofrece la emisora y el que ofrecen las emisoras competidoras, 
utilizando el algoritmo descrito en Sotelo et al. (2009).

\section{La recomendación de contenidos audiovisuales}

El campo de los recomendadores de contenidos audiovisuales ha tenido grandes avances en los últimos años. Su recomendación originalmente estaba orientada a consumidores individuales, incluso preocupándose de cómo adecuar el modelado de los telespectadores según casos particulares de la red disponible como la de un escenario de radiodifusión, Bjelica (2010). Pero últimamente se ha extendido hacia los grupos reflejando la realidad de que habitualmente el consumo de productos audiovisuales se da en grupos (familias, grupos de amigos, redes sociales...), como puede verse por ejemplo en Choonsung et al. (2009).

\subsection{El recomendador AVATAR}

AVATAR, Blanco et al. (2006), es el punto inicial del trabajo que se presenta aquí. Es un conocido recomendador basado en la inferencia de relaciones semánticas entre programas mediante técnicas de la web semántica. En su núcleo encontramos una ontología OWL que (i) describe los programas de televisión, (ii) los clasifica en jerarquías de contenido basadas en clasificaciones TV-Anytime, y (iii) la relaciona a otros programas a través de sus características semánticas (elenco, lugar de filmación, fechas...). Los perfiles de telespectador consisten en extractos de la ontología multidimensional de la televisión que contiene los programas que un individuo dado ha calificado en el pasado, cada uno de ellos con un índice numérico asociado, llamado DOI (Degree Of Interest) que cuantifica su interés por el contenido.

Esta realimentación para un contenido dado (el DOI) puede ser ingresada explícitamente por el telespectador o inferida a partir de mediciones indirectas, tales como el tiempo que ha permanecido mirando el programa. A partir del índice DOI de cada programa, nuestra aproximación infiere el nivel de interés del telespectador tanto en los atributos de este programa como en las clases bajo las cuales está categorizado en la ontología. Específicamente, los índices DOI del programa se propagan a través de las clases de la jerarquía y los atributos de los programas.

En suma, nuestros perfiles de telespectador almacenan la lista de programas de televisión que un telespectador ha visto en el pasado, y cada programa está asociado con un vector de valores conteniendo los índices DOI de (i) el programa, (ii) sus atributos, y (iii) sus clases en la ontología de la televisión.

La estrategia de recomendación adoptada en AVATAR combina dos de las técnicas de filtrado más populares. Se trata de un recomendador híbrido, ya que emplea tanto filtrado basado en contenido como filtrado colaborativo. Para decidir si un programa TP es sugerido a un determinado telespectador $U$ se utiliza:

- Filtrado basado en contenido: esta técnica sugiere TP a $U$ si es similar a contenidos que el televidente haya visto en el pasado y le hayan gustado.

- Filtrado colaborativo: Al contrario, el filtrado colaborativo sugiere el contenido TP a $U$ si este programa ha sido de interés a individuos con preferencias similares (sus vecinos).

\subsection{TV-Anytime}

En Sotelo et al. (2009) ideamos un modelo ontológico mejorado, que refleja el esquema de clasificación multidimensional de TVAnytime en la estructura jerárquica, añadiendo una rama a la ontología para cada uno de los esquemas de clasificación TV-Anytime más importantes (Intention, Format, Content e IntendedAudience). Esto mejora en gran medida la riqueza de la ontología comenzando por descripciones de contenido TV-Anytime, y permite mejorar las comparaciones de perfiles en múltiples dimensiones con el fin de calcular similitudes entre contenidos.

Encontramos en el rico conjunto de metainformación definido en TV-Anytime un modo fácil y normalizado para recoger en el receptor de televisión digital (el set top box, $S T B)$ los perfiles de los telespectadores y su historial de uso. De esta forma se facilita la 
interacción entre productos de distintos fabricantes.

Concretamente las cuatro clasificaciones utilizadas se refieren a:

- Intention: contiene términos que describen la intención del programa en su fase conceptual. Entre otros, describe si su propósito es entretener, informar, educar, promocionar otros contenidos (tráileres), vender productos... Incluso se puede clasificar el nivel de dificultad de un contenido destinado a educar.

- Format: contiene términos que describen el formato del programa. Por ejemplo, si es una actuación artística, una revista, un dibujo animado. Pero también otras características como si es un programa interactivo, en cuyo caso los niveles inferiores aportan detalles como si la interactividad es local, intermitente, o permanente, y en cada una de ellas todo el espectro posible.

- Content: contiene términos que describen la naturaleza o el género del programa o comercial. Puede referirse en términos generales si es un programa de ficción, informativo, deportivo, musical, entre otros. Es la clasificación más poderosa ya que en algunas ramas llega a tener cuatro niveles de profundidad. Para nuestro trabajo resulta la más importante ya que refleja el principal aspecto de interés del espectador.

- Intended Audience: contiene términos que describen la audiencia a la que va dirigido el programa, por ejemplo, por género, edad, grupo socioeconómico o nivel educativo.

En la Tabla 1 mostramos un extracto de la clasificación Content. Se observa que este esquema de clasificación que ofrece TVAnytime es muy amplio, con nueve grandes grupos de géneros para los contenidos, y jerárquico, con hasta cuatro niveles de profundidad. Puede apreciarse que constituye una clasificación muy rica para los contenidos audiovisuales.

\subsection{Recomendación para grupos}

Asimismo, en Sotelo et al. (2009) presentamos un nuevo algoritmo para otorgar recomendaciones a grupos, especialmente grupos heterogéneos, para los que no se habían logrado buenos resultados en el estado del arte según puede verse en Yu et al. (2006), de Campos et al. (2007), Shin et al. (2008) o Vildjiounaite et al. (2008). Tal como se dijo en la introducción, distinguimos entre grupos homogéneos y grupos heterogéneos, y realizamos recomendaciones adecuadas para cada clase de grupo. Con este algoritmo, y partiendo del DOI de los telespectadores en las clases de la ontología, AVATAR es capaz de predecir el Predicted DOI (PDOI) del grupo por un nuevo contenido.

Tabla 1 - Extracto de la clasificación Content.

\begin{tabular}{|c|c|}
\hline Código & Género \\
\hline 3.1 & NON-FICTION/INFORMATION \\
\hline 3.1 .1 & News \\
\hline 3.1 .1 .1 & Daily news \\
\hline$\ldots$ & $\ldots$ \\
\hline 3.1 .1 .10 & Cultural \\
\hline 3.1 .1 .10 .1 & Arts \\
\hline$\ldots \ldots$ & $\ldots \ldots$ \\
\hline 3.1 .1 .17 & Consumer affairs \\
\hline 3.1 .2 & Religion/Philosophies \\
\hline$\ldots \ldots$. & $\ldots \ldots \ldots$ \\
\hline 3.1 .4 & Arts \\
\hline 3.1 .4 .1 & Music \\
\hline ........ & ......... \\
\hline 3.1 .4 .12 & Showbiz \\
\hline 3.1 .5 & Humanities \\
\hline & \\
\hline 3.2 & SPORTS \\
\hline$\ldots \ldots$. & $\ldots \ldots .$. \\
\hline 3.4 & FICTION/DRAMA \\
\hline$\ldots$ & $\ldots$ \\
\hline 3.5 & AMUSEMENT/ENTERTAINMENT \\
\hline ....... & ........ \\
\hline 3.6 & Music \\
\hline$\ldots$ & $\ldots$ \\
\hline 3.7 & INTERACTIVE GAMES \\
\hline$\ldots \ldots$. & ........ \\
\hline 3.8 & LEISURE/HOBBY/LIFESTYLE \\
\hline$\ldots \ldots$ & $\ldots \ldots \ldots$ \\
\hline 3.9 & ADULT \\
\hline
\end{tabular}

Clasificamos el grupo $G$ como homogéneo o heterogéneo computando la correlación de los vectores de interés de los perfiles de telespectador de sus miembros. Definimos que dos telespectadores son vecinos si su correlación está sobre un cierto umbral (tienen preferencias similares). Cuando el grupo $G$ es homogéneo (todos los miembros son vecinos dos a dos), fusionamos los perfiles y computamos el interés del "telespectador virtual" resultante y que representa al grupo, utilizando el motor del AVATAR original. Cuando el grupo $G$ es 
heterogéneo, utilizamos filtrado colaborativo, buscando grupos similares (vecinos) a $G$ (los grupos vecinos tienen al menos un miembro vecino a cada miembro de $G$ ). Finalmente, recomendamos un nuevo contenido a $G$ si ha sido de interés a sus grupos vecinos.

\section{Escenario para la medición de audiencia}

El número de televidentes que integran la audiencia es sumamente grande, mucho mayor que los grupos con los que se ha trabajado en la recomendación para grupos. Por tanto, resulta natural que debamos realizar consideraciones especiales para tratarlo y para obtener predicciones certeras. Asumimos que trabajamos con un panel, es decir, una selección representativa de la audiencia, en la que los televidentes de los hogares que la integran son conscientes de que participan y están dispuestos a colaborar. En realidad, es concebible que en un futuro se reciba de parte de todos los telespectadores la información sobre su consumo de televisión, con los permisos correspondientes. Lo que aquí llamamos panel, en ese caso podría ser el total de la audiencia, totalizando miles o millones de hogares alimentando el sistema. Pensando en este escenario global, contemplaremos, en el análisis posterior, la posibilidad de simplificar los cálculos agrupando a los televidentes por intereses, segmentando la audiencia.

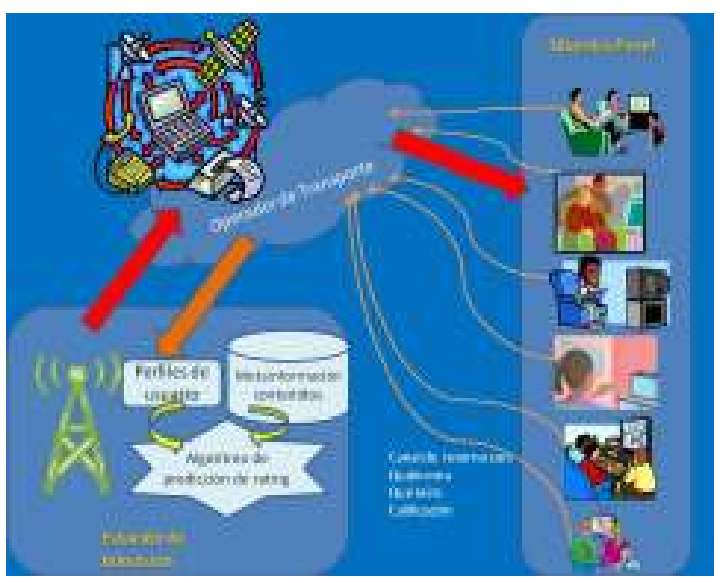

Figura 2 - Flujo de información para la predicción del rating
El marco tecnológico es el esquematizado en la Figura 2, en el que se aprecia que la clasificación de los contenidos TV-Anytime residirá solamente en el radiodifusor. EI STB no necesita entender las jerarquías TVAnytime ya que la recomendación (de hecho, la predicción de rating) se hará en la estación de televisión y no en el STB. Prevemos que cada dispositivo por el que los telespectadores miran la televisión cumple con los siguientes requisitos:

1. Capacidad de registrar las personas que están mirando la televisión.

2. Capacidad de que los televidentes califiquen el programa que han visto.

3. Canal de retorno por el que se podrán enviar al radiodifusor, cableoperador u otro actor, la información de los dos puntos anteriores recogida por el dispositivo.

El radiodifusor entrega la señal al operador de transporte (por ejemplo, el cableoperador o la empresa telefónica en el caso de IPTV) quien la transporta hasta el televidente. Los datos recogidos por los dispositivos de telespectador llegan nuevamente al radiodifusor en tiempo real a través del canal de retorno en los cablemódems o a través de IP en el caso de IPTV. Esta información alimenta la base de datos con los perfiles de telespectador que se almacena en la estación de televisión y la mantiene actualizada.

Partiendo de los perfiles de telespectador y de la metainformación de los contenidos, la estación de televisión es capaz de ejecutar los algoritmos con las extensiones para grupos descritos en Sotelo et al. (2009).

El perfil del telespectador $U_{j}$ consta de cuatro vectores que lo representan, cada uno de ellos referido a una de las cuatro jerarquías Intention, Format, Intended Audience y Content, con dimensiones $n^{I}, n^{F}$, $n^{I A}, n^{C}$ respectivamente, como se ve en la Ecuación 1.

Cada una de las dimensiones representa el interés del telespectador (el DOI) en cada una de las clases de las cuatro jerarquías representadas en nuestra ontología de la televisión. 


$$
U_{j}=\left\{\begin{array}{lr}
\left(v^{I}{ }_{j}[1], \ldots, v_{j}{ }_{j}\left[n^{I}\right]\right) & \text { Intention } \\
\left(v^{F}{ }_{j}[1], \ldots, v^{F}{ }_{j}\left[n^{F}\right]\right) & \text { Format } \\
\left(v^{I A}{ }_{j}[1], \ldots, v^{I A}{ }_{j}\left[n^{I A}\right]\right) & \text { Intended Audience } \\
\left(v^{C}{ }_{j}[1], \ldots, v^{C}{ }_{j}\left[n^{C}\right]\right) & \text { Content }
\end{array}\right.
$$

Ecuación 1

La estación de televisión también tiene la ontología que almacena y estructura la metainformación que describe los contenidos.

Sin embargo, la audiencia de una estación de televisión es un número muy grande. Dependiendo de si su alcance es local o nacional, la audiencia representa miles o millones de personas. Es necesario, por tanto, segmentarla.

\section{Modelado de la Audiencia: Segmentación en Subgrupos}

Nuestra estrategia será la de segmentar la audiencia en subgrupos relativamente homogéneos según sus intereses. En tal sentido, partiendo de la observación, podemos asumir que en la audiencia existen subgrupos homogéneos que comparten intereses comunes, que tal vez estén representados por grupos socioeconómicos, o por grupos de interés.

Atendiendo a ello, para simplificar la tarea de cálculo y representación del grupo, luego de buscar estos subgrupos homogéneos, sustituiremos los perfiles de sus integrantes por un perfil del subgrupo. Este procedimiento, llamado clustering, agrupa los puntos próximos en el espacio vectorial de los perfiles de telespectador, $y$, por tanto, cada punto es el vector de valoraciones de las categorías de cada telespectador del panel. Por tanto, la información que tendremos en el perfil del subgrupo obtenido será el vector de las valoraciones de las categorías. Este punto, que llamaremos centroide, representa un telespectador virtual equivalente al subgrupo.

Cada subgrupo tiene un número distinto de miembros, o, lo que es lo mismo, cada telespectador virtual representa una cantidad diferente de telespectadores. Por tanto, cuando se tomen decisiones a partir de los telespectadores virtuales, se debe asignar un peso relativo distinto a cada uno, proporcional al número de telespectadores reales que representa.

Existen diversos algoritmos para realizar el clustering en un problema de minería de datos (data mining), Berkhin (2006). Estos procesos se aplican sobre un conjunto muy grande de elementos en un espacio vectorial en el que se pueden medir distancias. Se agrupan (o crean clusters) y todos los elementos que pertenecen a un cluster son sustituidos por un elemento que los representa, su centroide, a efectos de poder procesarlos de manera simplificada. De otra forma, al ser muy grande la cantidad de elementos, resulta en una carga computacional muy alta.

Hemos utilizado el algoritmo $x$-means, descrito en Pelleg (2000), que supera ciertas limitaciones de otros algoritmos, y que ha sido empleado también por el recomendador de televisión descrito en "More Like This" or "Not for Me" , Bonnefoy (2007). Según consigna Delibašic (2009), el algoritmo utiliza una estrategia de partición mediante división binaria y tiene la ventaja que el telespectador no debe indicar el número $k$ de clusters resultantes, sino sólo un número mínimo y máximo de clusters. El propio algoritmo encontrará el $k$ más apropiado.

Con esta representación en subgrupos se logra reducir enormemente la cantidad de vectores de telespectador; de los $n$ telespectadores que forman parte de la muestra, se consigue trabajar con $k$ (que sin duda representa un número mucho menor), y así se hacen manejables los cálculos que implican nuestros algoritmos de predicción. De hecho, este procedimiento nos habilita a trabajar con el total de la audiencia si un día resultara factible contar con la participación de todos los televidentes. Los centroides obtenidos (telespectadores virtuales) los denotaremos por $V_{1}$ a $V_{k}$ y cada uno representa un conjunto de telespectadores con diferente cardinal, y están definidos por un vector de DOIs en las clases de la ontología.

En la Figura 3 puede verse un ejemplo simple del resultado de la aplicación del algoritmo, en el que los telespectadores están identificados por los puntos pequeños. 
Como se ve, resultan cuatro subgrupos, representados por cuatro telespectadores virtuales que están simbolizados por los cuatro puntos mayores.

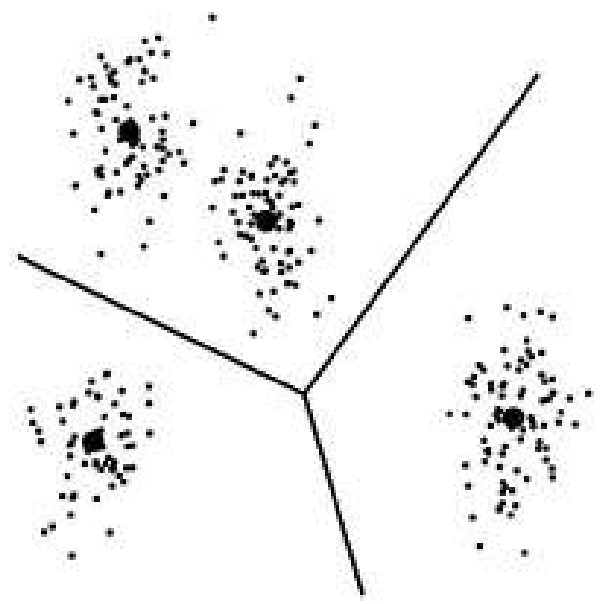

Figura 3 - Clustering de la audiencia en subgrupos homogéneos, imagen de Pelleg (2000).

En general, el número $k$ de subgrupos obtenidos dependerá de cada mercado en el que se implante el sistema, y se adecuará a las características de la audiencia en él. Las cotas superior e inferior para el número $k$ que debe definir el telespectador se configuran de acuerdo al conocimiento que se tenga sobre la audiencia. En nuestro contexto, la cota inferior será 1 para considerar el caso de que existiera una audiencia homogénea (podría ser el caso de cierto mercado reducido), y la superior 10 , para no tener un excesivo número de clusters.

Esta segmentación de la audiencia agrupa los telespectadores según sus preferencias, marcando una ventaja sobre la situación actual en que la audiencia se segmenta según datos demográficos, como el sexo, la edad o el nivel socioeconómico. No podemos negar que esto último tenga verosimilitud, y que lleve a buenas predicciones de audiencia, pero, evidentemente, la propuesta metodológica que presentamos representa una situación notablemente superior. Este procedimiento de clustering o agrupamiento de telespectadores es un procedimiento semántico, ya que los DOIs de las clases (las distintas componentes de los vectores del perfil de telespectador) se calculan empleando las características semánticas, que reflejan el interés de los telespectadores. Como el origen de los vectores de DOIs está relacionado con las características semánticas de los contenidos, el proceso de clustering proviene de un fundamento semántico que resulta en una aproximación novedosa e interesante. Por tanto, estamos seguros que la agrupación de los telespectadores se realiza en función de sus intereses, y no de estereotipos más o menos afortunados, lo que conduce a que la predicción del rating para un cierto contenido, del cual contamos con su clasificación en nuestra ontología, podrá ser hecha de manera más precisa.

\section{Generación de la Parrilla}

La selección de los programas a emitir es una tarea muy importante. La decisión depende de las características de la audiencia de la cadena de televisión, el día, la hora, los costos de los programas, la emisión de los radiodifusores competidores... Los gerentes de programación de los canales de televisión tienen que construir su parrilla de programación a transmitir eligiendo entre un cierto número de contenidos audiovisuales $\left(C_{1}\right.$ a $\left.C_{n}\right)$ que la estación tiene el derecho a emitir, en busca de la mejor distribución para aumentar los ingresos.

Vamos a exponer ahora nuestro algoritmo para ayudar a los gerentes con la programación. El objetivo es obtener la disposición de los programas en la parrilla de programación que maximiza el rating acumulado en toda la semana. Los datos de entrada serán el conjunto de contenidos $C_{1}$ a $C_{n}$ clasificados en la ontología junto con los programas ya emitidos, y el vector de los intereses de los telespectadores reales recibidos de los decodificadores de los panelistas (los integrantes de la muestra).

El primer paso consistirá en calcular, mediante el procedimiento de clustering, los telespectadores virtuales $\left(V_{1}\right.$ a $\left.V_{k}\right)$, que representan a toda la audiencia. A continuación, calculamos el PDOI para cada contenido $C_{i}$ para cada telespectador virtual utilizando el algoritmo de AVATAR para 
grupos. De este modo, se obtiene un indicador del interés de cada subgrupo de la audiencia para cada contenido disponible.

Como es habitual, se divide el día en cinco períodos diferentes de tiempo (Matutino, Mediodía, Vespertino, Prime Time y Trasnoche). Las mediciones reales muestran que la audiencia total es constante día tras día en el mismo período de tiempo, por lo que se considera el mismo público total en cada período de tiempo todos los días de la semana. Como se puede observar en la Figura 4, se considera una audiencia constante total $A_{j}$ para cada período a efectos de simplificar la exposición. Por lo tanto, $A_{1}$ representará a la audiencia total en la franja del horario Matutino, y $A_{5}$ de la audiencia total del Trasnoche.

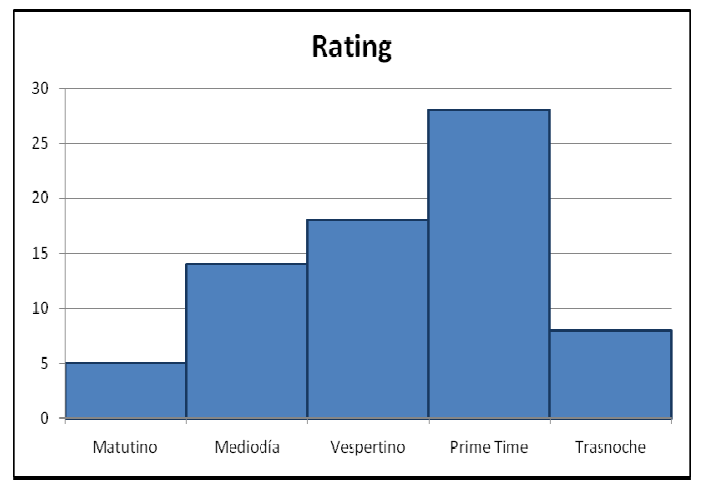

Figura 4 - Distribución de la audiencia en un día típico.

Recordemos que los telespectadores $V_{1}$ a $V_{k}$ son los telespectadores virtuales que representan los subgrupos en que hemos dividido nuestra audiencia, basándonos en sus perfiles de telespectador, de acuerdo a sus intereses y no a sus clasificaciones socioeconómicas. Cada uno de ellos representa a un número de telespectadores que ha sido agrupado por el algoritmo $x$ means. Presumiblemente, las personas que forman parte de cada uno de estos subgrupos, no tienen la misma asiduidad para ver televisión, ni lo hacen en las mismas franjas horarias.

La audiencia de cada uno de los horarios está compuesta por integrantes de cada uno de los subgrupos, pudiendo determinarlo el radiodifusor de acuerdo a la información histórica de que dispone. Podemos entonces hablar para todos los $k$ subgrupos de una audiencia para cada horario tal como aparecen en la Tabla 2:

Tabla 2. Audiencia de cada grupo en cada franja horaria.

\begin{tabular}{|c|c|c|c|}
\hline Audiencia en horario Matutino & $\mathrm{A}_{11}$ & $\ldots \ldots$. & $\mathrm{A}_{1 \mathrm{k}}$ \\
\hline Audiencia en horario Mediodía & $\mathrm{A}_{21}$ & $\ldots \ldots$ & $\mathrm{A}_{2 \mathrm{k}}$ \\
\hline Audiencia en horario Vespertino & $\mathrm{A}_{31}$ & $\ldots \ldots$ & $\mathrm{A}_{3 \mathrm{k}}$ \\
\hline Audiencia en Prime Time & $\mathrm{A}_{41}$ & $\ldots \ldots$. & $\mathrm{A}_{4 \mathrm{k}}$ \\
\hline Audiencia en horario Trasnoche & $\mathrm{A}_{51}$ & $\ldots \ldots$. & $\mathrm{A}_{5 \mathrm{k}}$ \\
\hline
\end{tabular}

También asumimos que hay $E$ estaciones de televisión competidoras para las que conocemos la parrilla de programación (tentativa). Se trata de una simplificación plausible, ya que los ejecutivos de la televisión conocen los programas que ha adquirido o producido la competencia, y conocen su historial de programación.

El rating que tendrá un contenido $C$ emitido por nuestra emisora en una franja horaria $j$ lo estimaremos de acuerdo a la Ecuación 2:

$$
R_{j}(C)=\sum_{i=1}^{k} \frac{D O I(C)_{\mathrm{i}}}{D O I(C)_{\mathrm{i}}+\sum_{l=1}^{E} D O I\left(C_{l}\right)_{\mathrm{i}}} \cdot A_{\mathrm{ji}}
$$

\section{Ecuación 2}

donde $A_{j i}$ es el número de televidentes del subgrupo del telespectador virtual $V_{i}$ que miran televisión en el período $j$, y $C_{1}, C_{2} \ldots C_{E}$ los contenidos emitidos por la competencia.

Consideremos la fórmula de la Ecuación 2, donde el rating estimado se compone de la suma de las porciones de audiencia de los distintos subgrupos que estimamos será capaz de captar nuestra estación. Recordemos que estamos estimando para el horario $j$, por ello es que aparece dicho sufijo en ambos lados de la ecuación. El resultado es el rating previsto en ese horario. La sumatoria se aplica sobre los $k$ subgrupos de interés en los que hemos dividido nuestra audiencia. En el caso del horario $j$ que nos ocupa, la audiencia $A_{j}$ estará compuesta por la suma de $A_{j 1}+A_{j 2}$ $+\ldots+A_{j k}$. Si fuéramos la única emisora que transmite en el mercado, $E$ sería cero, y el rating esperado de $C$ en el horario $j$ será $A_{j}$, la audiencia total disponible, como era de esperar. 
Cuando hay otras emisoras $(E \neq 0)$, estimamos qué porción de los integrantes de $A_{j i}$ vendrán a nuestra emisora y los sumamos. Este ratio para el grupo $i$ se computa dividiendo el interés del grupo por nuestro contenido por el interés total de ese grupo en todos los contenidos disponibles en esa franja horaria.

El subíndice $i$ indica el subgrupo de interés (representado por el telespectador virtual $V_{i}$ ). Utilizando este factor estimaremos cuánta audiencia captará la emisora mediante el programa $C$, de la total disponible $A_{j i}$ en el horario $j$ para el subgrupo $i$. Podemos ver que en el numerador tenemos el grado de interés que despierta nuestro contenido $C$ para la audiencia $A_{j i}$. Mientras que en el denominador tenemos la suma de los intereses que despiertan en dicha audiencia cada uno de los contenidos ofrecidos simultáneamente por las otras emisoras.

Ilustraremos la Ecuación 2 con algunos ejemplos.

Si hubiera $E$ emisoras (con $E \neq 0$ ) además de la nuestra, y su contenido fuera extremadamente equivalente al nuestro $y$ despertara, por tanto, el mismo interés, $\operatorname{DOI}(C)_{i}=\operatorname{DOI}\left(C_{l}\right)_{i}$ para todos los subgrupos, esperaríamos que el rating predicho para nuestra emisora fuera la $(E+1)$-ésima parte de la audiencia total disponible en el horario. En efecto, obtenemos la Ecuación 3:

$R_{j}(C)=\sum_{i=1}^{k} \frac{D O I(C)_{\mathrm{i}}}{\operatorname{DOI}(C)_{\mathrm{i}}+\sum_{l=1}^{E} \operatorname{DOI}\left(C_{1}\right)_{\mathrm{i}}} \cdot A_{\Downarrow}=\sum_{i=1}^{k} \frac{1}{1+E} \cdot A_{\Downarrow}=\frac{1}{1+E} \cdot A_{\jmath}$

Ecuación 3

Por el contrario, supongamos que nuestro contenido $C$ es de sumo interés para el subgrupo $1\left(\mathrm{DOI}(\mathrm{C})_{1}=1\right)$, y los contenidos ofrecidos por la competencia tienen un grado de interés nulo $\left(\operatorname{DOI}\left(C_{1}\right)_{1}=0\right)$ para dicho subgrupo. Esperamos que el rating sea al menos $A_{j 1}$. A esa audiencia podrá sumarse una porción de los integrantes de los subgrupos restantes, proporcional a su interés, tal como lo ilustra la Ecuación 4.

Asumiremos por simplicidad que los $n$ programas disponibles y los $m$ "huecos horarios" de que disponemos en la semana son todos de la misma duración. Así, habrá $m_{j}$ huecos horarios en el período $j$ durante la semana. Por lo tanto, para cada selección de $m$ programas llenando los $m$ huecos horarios, podemos computar el rating acumulado de esa distribución de acuerdo a la Ecuación 5. De esta forma, computamos todas las distintas posibilidades $(n ! /(n-m) !)$ de los $n$ programas en los $m$ huecos temporales y la distribución que tiene el mayor rating predicho será la que se recomiende al gerente de programación de la estación de TV.

$$
\begin{gathered}
R_{j}(C)=\frac{1}{1+\sum_{l=1}^{E} 0} \cdot A_{\mathrm{j} 1}+\sum_{i=2}^{k} \frac{D O I(C)_{\mathrm{i}}}{D O I(C)_{\mathrm{i}}+\sum_{l=1}^{E} D O I\left(C_{\mathrm{l}}\right)_{\mathrm{i}}} \cdot A_{\mathrm{ji}} \geq A_{\mathrm{j} 1} \\
R_{A}=\sum_{j=1}^{5} \sum_{p=1} R_{j}\left(C_{p}\right) \\
\text { Ecuación } 4 \\
p=m_{j}
\end{gathered}
$$

Los programas a emitir, ya sean adquisiciones o producidos por la propia emisora, siempre tienen un coste. Cada contenido $C_{i}$ tiene un coste $P_{i}$, por tanto para cada distribución de programas $A$ podemos calcular el coste total $P_{A \text {-TOTAL }}$ como la suma de los costes de todos los programas utilizados en la distribución $A$.

Las emisoras comerciales deben estar pendientes de sus ingresos, pero más que nada de sus beneficios, es decir la relación entre los ingresos y los egresos. Los ingresos están directamente relacionados con el rating, sin embargo los costes no lo están.

Cabe introducir el siguiente indicador, que será de interés al encargado de programación del canal, en conjunto con el encargado de administración, y más precisamente, para la gerencia general que debe estar pendiente de los beneficios de la empresa, mirando tanto los ingresos, y por lo tanto el rating, como los egresos, entre ellos los costes de los programas.

Definimos la Eficiencia de la distribución de programas $A$ en la Ecuación . 


$$
E_{A}=\frac{R_{A}}{P_{A} T O T A L}
$$

Ecuación 6

Dicha Eficiencia refleja la relación rating obtenido en la semana, a costes en que se incurre. Calculándola para todas las posibles distribuciones, existirá una distribución $A_{\max }$ para la que la $E_{A m a x}$ sea máxima, representando la mejor relación rating a costes.

\section{7 ¿Qué emitir a continuación?}

\subsection{Algunas características de los programas en vivo}

Usualmente los contenidos de la televisión en vivo son decididos por el productor del programa en tiempo real en el control de emisión, normalmente basándose en su intuición. El productor de un programa es quien decide el contenido dentro del programa. Mientras que el encargado de programación crea la grilla semanal basándose en las características de los programas a emitirse en un trabajo que podríamos denominar como fuera de línea, el productor, también conociendo las características de su programa, habitualmente tiene opciones para alterar su contenido o estructura mientras está siendo emitido. Ellos deben decidir, conociendo la audiencia que tiene su estación de televisión en un determinado momento y lo que está siendo emitido por las emisoras competidoras y sus ratings respectivos, qué emitir a continuación entre ciertas opciones, dentro de un mismo programa o a la finalización del mismo. Podemos pensar en las siguientes decisiones que corresponden al productor de un programa durante su emisión: (i) en un reality show a qué personaje seguir, (ii) en una carrera de Fórmula 1 si ocuparse del puntero o de otros dos corredores que protagonicen un duelo importante o de un corredor que represente un interés especial por ejemplo por su nacionalidad, (iii) en un noticiero decidir si seguir con las noticias de carácter policial o cambiar a las noticias deportivas, (iv) en un partido de fútbol si seguir a una estrella de un equipo o nacionalidad particular, (v) si conviene cortar la exposición de un invitado porque el rating baja, (vi) a qué invitado darle la palabra, (vii) si seguir en vivo desde el estudio con un debate, o reproducir una nota pregrabada.

La selección debe hacerse pensando en el televidente. Una buena selección es vital para que el programa tenga buena audiencia y para que el canal de televisión tenga buenos ingresos. La predicción de audiencia se realiza a menudo basándose en criterios tales como mediciones de audiencia anteriores, la clasificación estereotipada de la audiencia (sobre la base de información demográfica) y la intuición del productor.

La segunda aplicación que presentamos aquí es un algoritmo que recomienda, en tiempo real, qué contenido, de entre un conjunto disponible, una estación de televisión debe emitir en un momento determinado, teniendo en cuenta las mediciones de audiencia en línea en tiempo real y los perfiles de los telespectadores de su audiencia. En la actualidad, estos sistemas de medición en línea en tiempo real comienzan a ser implementados con éxito como por ejemplo el descrito por Crivellaro et al. (2008).

Hay ciertos datos que son conocidos de antemano a través del sistema de medición. Sabemos en tiempo real que la audiencia total es $A$ y nuestra audiencia es $a$, constituida por los consumidores con perfiles virtuales $U_{i}$. La audiencia de los competidores es $A-a$, constituido por los consumidores con perfiles virtuales $V_{i}$. Ambos $U_{i}$ y $V_{i}$ se obtienen por el procedimiento de clustering que ya se ha descrito en la sección 5 .

Para maximizar el impacto de cada programa, y para hacer más realista nuestro algoritmo, debemos tomar en cuenta el hecho de que la gente tiende a permanecer en la misma emisora que está viendo, sin hacer zapping. Por ejemplo, si el televidente tiene disponible un contenido alternativo al que está viendo, ambos con el mismo PDOI, no cambiará de canal. Es así que debemos:

- Mantener nuestra audiencia. Para ello tendremos cuidado de que ninguno de ellos esté disgustado con lo que emitamos a continuación. 
- Ganar parte de la audiencia de la competencia. Para ello ofreceremos contenido muy atractivo a algunos de ellos.

\subsection{Posibles estrategias agregación de intereses}

de

A efectos de estimar el interés de un grupo de televidentes por un contenido pueden utilizarse distintas estrategias para agregar o tener en cuenta en conjunto los intereses de los distintos integrantes de un grupo. Estas han sido estudiadas en diversos trabajos de Masthoff (2002, 2004) y sintetizadas en Jameson (2007).

\section{A. Maximizar la Satisfacción Promedio}

Una primera posibilidad es la de estimar la satisfacción del grupo mediante el promedio del interés que despierta el contenido entre cada uno de los usuarios como se ve en la Ecuación 7:

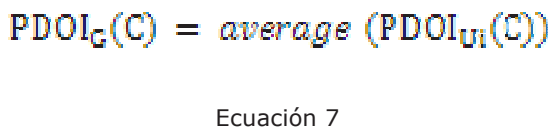

Esta estrategia de agregación simplemente promedia el interés que despierta el contenido entre todos los usuarios. Es una estrategia justa. Seguirla y recomendar el máximo PDOI para el grupo obtenido por este método, tiene la ventaja de que maximiza la satisfacción general del grupo.

\section{B. Minimizar Miseria}

Si hay algún miembro del grupo a quien le disgusta en extremo el contenido $C$, puede que esté en total desacuerdo con el resultado de la estrategia anterior. Así, el grupo podría considerar que elegir la opción que resulta de la estrategia del promedio de satisfacción, no es un buen resultado ya que está haciendo miserable a uno o más de sus integrantes. En ese caso podría tomar otra alternativa para minimizar la miseria, que puede consistir en no tomar ninguna opción que para cualquiera de los individuos caiga debajo de un determinado umbral. Eliminadas esas alternativas, con las restantes efectuar el promedio para maximizar satisfacción.

\section{Asegurar cierto grado de Equidad}

La estrategia anterior puede llevar a una solución mediocre, en la que todos tengan un grado bajo de satisfacción. Puede que como disgustan a unos miembros, se eliminen alternativas que sean de alto interés para otros, y que las que sobrevivan no sean de alto interés para nadie. Buscando atemperar este hecho, pero manteniendo la prioridad por la uniformidad de los intereses, al calcular la agregación de las calificaciones individuales, en la Ecuación 8 incluimos un término de penalización que refleje la cantidad de variación entre los ratings predichos:

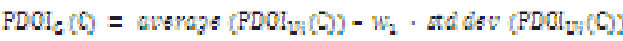

Ecuación 8

Donde $w_{1}$ es un peso que refleja la importancia relativa de la Equidad, y std dev es la desviación estándar de los grados de interés predichos para el contenido $C$ para todos los usuarios virtuales $U_{i}$.

Es decir, std $\operatorname{dev}\left(\mathrm{PDOI}_{\mathrm{Ui}}(\mathrm{C})\right)$ nos muestra cuán dispares son los grados de interés predichos entre los distintos usuarios. En esta estrategia, dado un contenido C, computamos el promedio de los intereses predichos para todos los usuarios, y le restamos la desviación estándar ponderada con $w_{1}$. De esta forma, el grado de interés que prevemos para el grupo para el contenido $C$, aunque el promedio sea igual en dos casos, será menor cuando el contenido despierta un interés muy dispar entre los miembros del grupo. Esta situación refleja que habría miembros muy disgustados si se elige dicha opción. Por eso se la penaliza, disminuyéndola. En el caso contrario, mismo promedio pero menor desviación estándar, significa que el contenido propuesto, generará la misma aceptación promedio en el grupo, y que todos los miembros lo aprecian cerca del 
promedio, es decir, no genera calificaciones extremas, extremo gusto o extremo rechazo. Esta estrategia, favorece este último tipo de situaciones.

\section{Asegurar cierto grado de Placer}

Contrariamente a lo expuesto en las estrategias precedentes, especialmente en las B y C, puede ser deseable asegurar que a algunos de los miembros del grupo les guste mucho la recomendación. Es decir, que independientemente de que algunos se sientan desconformes con la recomendación, existan miembros que la valoren muy positivamente, y resulten muy satisfechos. Si es así, agregaríamos la presente variante para asegurar que al menos una parte del grupo siente una atracción fuerte por los contenidos recomendados. La agregación de las calificaciones individuales predichas podría incluir un término de bonificación que refleje la cantidad de variación entre las calificaciones pronosticadas, como en la Ecuación 9:

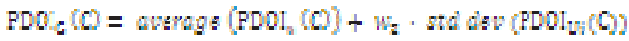

\section{Ecuación 9}

Donde $w_{2}$ es un peso que refleja la importancia relativa del Placer, y std dev nuevamente es la desviación estándar de los grados de interés predichos para el contenido $C$ para todos los usuarios $U_{i}$. Es una estrategia opuesta a la de "Asegurar cierto grado de Equidad". En esta nueva estrategia, favorecemos que aunque el promedio sea igual en dos casos, exista una gran diversidad de grados de interés predichos para cada individuo. Lo cual implica que hay una porción de los miembros del grupo a los cuales les gusta mucho el contenido propuesto.

\subsection{La Estrategia de Predicción}

Nos centramos ahora en la estrategia de predicción para elegir el contenido a emitir a continuación. Buscando maximizar el rating para nuestra emisora, debemos tratar de mantener nuestra audiencia intentando ganar algo de la audiencia de la competencia. Recordemos que debemos tener en cuenta el hecho de que la gente tiende a permanecer en la emisora, sin cambiar de estación, continuando con el programa que está viendo. Por ello buscaremos que el contenido a ofrecer a continuación sea del agrado de nuestra audiencia, sin despertar insatisfacciones mayores, mientras que para captar audiencia de la competencia trataremos de ofrecer contenidos de alto interés para cierta parte de ella.

Teniendo en cuenta la primera directiva, dado un nuevo contenido propuesto $C_{j}$, estimamos el interés de nuestra audiencia por él mediante la Ecuación 10 tratando de asegurar cierto grado de equidad:

$$
\begin{gathered}
\operatorname{PDOI}_{K E E P}(C j)=\frac{a}{A} \cdot(\boldsymbol{a v g}(\operatorname{PDOI} U \boldsymbol{i}(\boldsymbol{C} j))-\boldsymbol{w 1} \cdot \operatorname{std} \operatorname{dev}(\operatorname{PDOI} U \boldsymbol{i}(C j))) \\
\text { Ecuación } 10
\end{gathered}
$$

Con esta fórmula, intentamos beneficiar contenidos que no disgustarán a nuestros televidentes. Calculamos el promedio de los PDOIs para cada telespectador, y restamos la desviación estándar (ponderada), para beneficiar los contenidos que nadie odia. De esta forma, penalizamos los contenidos que presentan una alta desviación estándar en los PDOIs; dichos contenidos pueden ser no adecuados para algunos televidentes.

Cuando consideramos la audiencia de la competencia, debemos beneficiar contenidos que sean atractivos a una porción de la audiencia de la competencia. Utilizamos la estrategia "Asegurar cierto grado de Placer", tratando de que nuestro programa se vuelva muy atrayente para alguna gente de la que está mirando la competencia. Por tanto, se suma la desviación estándar (ponderada) al promedio de los PDOIs, como puede verse en la Ecuación 11:

$$
\text { PDOI }_{G A I N}(C j)=\frac{A-\boldsymbol{a}}{A}(\operatorname{avg}(\text { PDOI Vi }(C j))+w 2 \cdot s t d \operatorname{dev}(\text { PDOI Vi }(C j))
$$

$$
\text { Ecuación } 11
$$

De esta forma, promovemos grupos con una alta desviación estándar porque eso significa que algunos televidentes de la audiencia de la competencia estarán muy atraídos por el contenido que planeamos ofrecer. Esto es llamado asegurar cierto grado de placer en 
el campo de técnicas de agregación de grupos. Podemos esperar que algunos de ellos cambien a nuestro canal. Incluimos los parámetros w1 y w2 para ajustar el peso de la desviación estándar en cada término. Finalmente, combinamos ambos componentes de acuerdo a la Ecuación 12, usando el parámetro $\beta$ para priorizar nuestra audiencia o la de la competencia:

$$
\begin{aligned}
& \boldsymbol{P D O I}_{A U D}(\boldsymbol{C} \boldsymbol{j})=\boldsymbol{\beta} \cdot \boldsymbol{P D O I _ { K E E P }}(\boldsymbol{C} \boldsymbol{j})+(1-\boldsymbol{\beta}) \cdot \boldsymbol{P D O I _ { G A I N }}(C \boldsymbol{j}) \\
& \text { Ecuación } 12
\end{aligned}
$$

El contenido con el PDOI ${ }_{\mathrm{AUD}}$ más alto será el recomendado a ser emitido a continuación.

\section{Conclusiones}

En este trabajo ha sido presentado un método para predecir el interés del público de una estación de televisión (modelado como un grupo de espectadores) por un contenido audiovisual. Nos permite hacer recomendaciones precisas a los gerentes de televisión cuando planifican la parrilla de emisión de la semana y cuando deciden en tiempo real cómo debe evolucionar su programa que mejoran las técnicas actuales de predicción, considerando las preferencias reales de la audiencia en lugar de sólo la información demográfica. La selección es la que predice los ratings más altos, y por tanto, mejores ingresos por publicidad.

La base del procedimiento es un recomendador de contenidos para grupos, basado en inferencia semántica de las relaciones entre los contenidos y las preferencias de los telespectadores. Los resultados proporcionan ayuda a los ejecutivos de la televisión en dos escenarios diferentes: (i) la construcción de la parrilla de programación semanal, y (ii) la toma de decisiones en tiempo real sobre la evolución de los programas en vivo. Para hacer frente a restricciones en tiempo real, el público se ha dividido en subgrupos y sustituido por televidentes virtuales utilizando técnicas de clustering.

A lo largo del proyecto, han sido ampliamente utilizadas metainformación y clasificaciones TV-Anytime para garantizar la disponibilidad de las descripciones de contenido suficientemente detalladas, y la viabilidad de mecanismos compatibles para anotar y recoger los perfiles de los telespectadores.

\section{Referencias}

ÁlVAREZ, F., MARTÍN, C.A., ALLIEZ, D., ROC, P.T., STECKEL, P., MENÉNDEZ, J.M., CISNEROS, G., JONES, S.T.; Audience measurement modeling for convergent broadcasting and IPTV networks; IEEE Transactions on Broadcasting, vol 55, n², june 2009

ANG, I., Desperately Seeking the Audience, Routledge (April 12, 1991), p. 40

BERKHIN P (2006) A survey of clustering data mining techniques. In: Kogan J, Nicholas C, Teboulle M (eds); Grouping multidimensional data. Springer, Berlin-Heidelberg, pp 25-71.

BJELICA, M.; "Towards TV recommender system: experiments with user modeling," Consumer Electronics, IEEE Transactions on , vol.56, no.3, pp.1763-1769, Aug. 2010

BLANCO, Y.; PAZOS, J.; LÓPEZ, M.; GIL, A.; RAMOS, M.; AVATAR: an improved solution for personalized TV based on semantic inference, IEEE Transactions on Consumer Electronics, vol. 52, 2006.

BONNEFOY, D. AND BOUZID, M. AND LHUILLIER, N.; MERCER, K.; "More Like This" or" Not for Me": Delivering Personalised Recommendations in Multi-user Environments; Lecture Notes in Computer Science, vol 4511, pp 87; 2007; Springer

CHOONSUNG SHIN; WOONTACK WOO; "Socially aware tv program recommender for multiple viewers," Consumer Electronics, IEEE Transactions on , vol.55, no.2, pp.927-932, May 2009 
CRIVELlARO, A.; ALMEIDA, M.; The Real Time Audience System, One Minute or Nothing; International Conference on Automated solutions for Cross Media Content and Multi-channel Distribution, 2008. AXMEDIS '08.

DE CAMPOS, L.M. FERNANDEZ-LUNA, J.M. HUETE, J.F. RUEDA-MORALES, M.A., Univ. de Granada, Granada; Group Recommending: A methodological Approach based on Bayesian Networks; IEEE 23rd International Conference on Data Engineering Workshop, 2007.

DELIBAŠIC, B., KIRCHNER, K., RUHLAND, J., JOVANOVIC, M., VUKICEVIC, M.; Reusable components for partitioning clustering algorithms; Springer; Artif Intell Rev (2009) 32:59-75; DOI 10.1007/s10462-0099133-6

GENSCH, D., SHAMAN, P.; Models of Competitive Television Ratings; Journal of Marketing Research, Vol. 17, No. 3 (Aug., 1980), pp. 307-315; Published by: American Marketing Association

JAMESON, A.; SMYTH, B.; Recommendation to Groups, chapter The Adaptive Web: Methods and strategies of web personalization, pp. 596-627. Springer, 2007.

MASTHOFF, J.; University of Brighton; Group Modeling: Selecting a Sequence of Television Items to Suit a Group of Viewers; User Modeling and User-Adapted Interaction 14: 37-85, 2004

MASTHOFF, J.; Modeling a group of television viewers. In proceedings of the Future tv: Adaptive instruction in your living room workshop, associated with ITS02; 2002

PELLEG, D., MOORE, A.: X-means: Extending K-means with Efficient Estimation of the Number of Clusters. In: Proceedings of the Seventeenth International Conference on Machine Learning, pp. 727-734 (2000)

SHIN, C.; DEY, A.; WOO, W.; Mixed-Initiative Conflict Resolution for Context-aware Applications; UbiComp'08, September 21-24, 2008.

SOTELO, R.; BLANCO, Y.; LÓPEZ, M.; GIL, A.; PAZOS, J. J.; TV Program Recommendation for Groups based on Muldimensional TV-Anytime Classifications, IEEE Transactions on Consumer Electronics, Feb 2009

VILDJIOUNAITE, E. AND KYLLÖNEN, V. AND HANNULA, T. AND ALAHUHTA, P.; Unobtrusive Dynamic Modelling of TV Program Preferences in a Household; Proceedings of the 6th European conference on Changing Television Environments; p. 91; 2008; Springer.

YU, Z., ZHOU, X., HAO, Y., GU, J.: TV program recommendation for multiple viewers based on user profile merging. User Modeling and User-Adapted Interaction 16(1) (2006) 63-82.

Recebido em maio de 2011

Aprovado para publicação em junho de 2011

\section{Rafael Sotelo}

Facultad de Ingeniería de la Universidad de Montevideo (FIUM). Doctor en Ingeniería Telemática por la Universidad de Vigo, MBA por el IEEM/UM, Ingeniero Electricista por la Universidad de la República; Montevideo - Uruguay. E-mail: rsotelo@um.edu.uy

\section{Alberto Gil Solla}

Departamento de Ingeniería Telemática de la Universidad de Vigo (DET-UVIGO). Es Doctor en Ingeniería Telemática e Ingeniero en Telecomunicación por la Universidad de Vigo; Vigo - España. E-mail: agil@det.uvigo.es 